\title{
An evaluation of the cost-effectiveness of using CHROMagar for yeast identification in a routine microbiology laboratory
}

\author{
S. AINSCOUGH and C. C. KIBBLER
}

Department of Medical Microbiology, Royal Free Hospital School of Medicine, Pond Street, London NW3 $20 G$

\begin{abstract}
CHROMagar, a chromogenic differential culture medium, is claimed to facilitate the isolation and presumptive identification of certain clinically important yeast species, e.g., Candida albicans. This study evaluated the cost-effectiveness and time advantage of using it in comparison with Sabouraud dextrose agar (SDA). Three possible pathways, each of which included the use of one or both media, were compared in a routine laboratory. A total of 21 yeast isolates was cultured from 298 clinical samples from neutropenic and AIDS patients. An overall sensitivity of $95.2 \%$ was observed for each medium and primary isolation on CHROMagar was found to be $100 \%$ sensitive and $100 \%$ specific for $C$. albicans. For identification purposes, after initial culture the use of CHROMagar provided the most economical and least time-consuming method. Direct inoculation on to CHROMagar is recommended for blood cultures when yeast cells are seen on microscopy and where early appropriate therapy is imperative.
\end{abstract}

\section{Introduction}

In routine diagnostic laboratories Sabouraud dextrose agar (SDA) is widely used for the isolation of all yeast species from clinical specimens [1]. SDA is a selective medium supporting the growth of most pathogenic fungi encountered in clinical samples. In some laboratories an indicator dye (bromocresol green) is added to the SDA medium, producing a green solid agar that allows strain differentiation. The SDA agar is usually incubated for $48 \mathrm{~h}$ to isolate yeasts from a clinical sample, although Cryptococcus neoformans may require several weeks.

Candida albicans is the yeast species isolated most often from clinical material [2,3] and is frequently of clinical importance. However C. krusei and C. glabrata are now being encountered with increasing frequency, and may be associated with the prophylactic use of fluconazole $[4,5]$, to which they are less susceptible. Therefore, identification has important therapeutic implications. Mixed cultures containing these organisms might also explain problems with therapy. However, epidemiological studies have shown mixed cultures containing $C$. albicans in $<10 \%$ of samples [6].

Received 18 Sept. 1997; accepted 7 Nov. 1997.

Corresponding author: Dr C. C. Kibbler.
Most clinical laboratories use the formation of a germtube in serum [7] as the initial test to differentiate $C$. albicans from other yeast species. This is then followed by more time-consuming and expensive biochemical identification [2] usually in the form of a commercially available kit. To fully identify a yeast may take up to $72 \mathrm{~h}$ from primary isolation of the organism.

CHROMagar Candida (CA), a yeast differential and selective medium, allows the recognition of mixed yeast cultures in clinical specimens [3]. The medium permits the presumptive identification of $C$. albicans from other Candida spp. Yeast populations are differentiated by colonial morphology and colours which are generated by a chromophore in the agar [3]. C. albicans strains produce $\beta$-N-acetylgalactosaminidase, which interacts with the chromophore (chromogenic hexosaminidase substrate) incorporated into the agar, and with incubation for $48 \mathrm{~h}$ produces green colonies, characteristic of all C. albicans isolates [2]. Other Candida spp. can also be differentiated, e.g., $C$. tropicalis produces purple colonies.

The objective of this study was to examine the use of $\mathrm{CA}$ as the primary or follow-up medium for the culture and identification of yeasts in a routine laboratory. The cost and time required to determine the full identification of a yeast isolate cultured from routine clinical specimens were studied in three different process pathways. 


\section{Materials and methods}

The investigation was done over 4 weeks on throat, mouth, oesophageal and stool samples taken from leukaemic and AIDS patients.

Sabouraud Dextrose Agar (Unipath, Basingstoke), and CHROMagar (CHROMagar, Paris, France) were prepared following the manufacturers' instructions, in petri dishes holding $15-\mathrm{ml}$ volumes of the liquid agar; 5-ml volumes of CA were prepared in the same manner but poured in 5-ml petri dishes. Plates prepared from both media were stored at $4^{\circ} \mathrm{C}$ until used.

In the routine microbiology laboratory, 298 clinical specimens from both sets of patients were inoculated on to one quarter of an SDA plate and one quarter of a CA plate and incubated aerobically for $24 \mathrm{~h}$ at $37^{\circ} \mathrm{C}$. If cultures were negative, plates were incubated for a further $24 \mathrm{~h}$ at $37^{\circ} \mathrm{C}$. Colony counts were performed on all positive isolates. Three possible pathways for identifying the positive yeast isolates were examined and are illustrated in Figs. 1, 2 and 3. The cost of labour for each pathway was determined from the average (UK) salary of a biomedical scientist. The costs of testing germ-tube production, culture of a swab specimen and performing an API (bioMerieux) identification test were calculated with Welcan units [8]. Inoculation of a swab specimen takes 3.00 units, a germ tube 5.00 units and an API test 4.00 units.

\section{Results}

Twenty-one yeast isolates were cultured from the 298 clinical samples. Some of the isolates were cultured from the same patient, as repeat specimens were often examined to detect the clearance of the organism cultured in primary specimens. Mixed fungal cultures were not obtained with either selective medium. Each medium failed to support the growth of one isolate (cultured on the other), giving an overall sensitivity of $95.2 \%$ for each medium.

The germ-tube test was found to have a sensitivity and specificity of $84.6 \%$ and $100 \%$, respectively. The growth of green colonies on CA was $100 \%$ sensitive and $100 \%$ specific for $C$. albicans. Table 1 shows the yield and characteristics of each yeast isolate cultured. Fig. 1 illustrates process pathway 1, showing the stages taken in identifying a yeast isolate by traditional methods. Fig. 2 shows the stages in identifying a yeast isolate when the SDA plate was used for primary inoculation and CA for identifying the $C$. albicans isolates. Fig. 3 illustrates the process pathway when CA was used for primary inoculation and for identification of any $C$. albicans isolates.

Table 2 illustrates the cost of labour, materials and minimum time taken to identify $C$. albicans and Candida spp. by pathways 1,2 and 3 .

\section{Discussion}

The yield (i.e., the number of colonies) of yeast strains on CA was greater than on SDA, as found in a study by Baumgartner et al. [9], which also demonstrated a detection rate of yeast on CA $20 \%$ higher than on SDA. However, there was no difference in the sensitivity (i.e., detection rate) of $\mathrm{CA}$ and SDA for primary culture, in keeping with other studies $[2,3]$.

Table 1. Yield and characteristics of 21 yeast isolates cultured from clinical specimens inoculated on to Sabouraud dextrose agar (SDA) and CHROMagar (CA)

\begin{tabular}{|c|c|c|c|c|c|c|c|}
\hline $\begin{array}{l}\text { Isolate } \\
\text { no. }\end{array}$ & Specimen & $\begin{array}{l}\text { CA } \\
\text { colour }\end{array}$ & $\begin{array}{c}\mathrm{CA} \\
\text { growth }\end{array}$ & $\begin{array}{l}\text { SDA } \\
\text { colour }\end{array}$ & $\begin{array}{l}\text { SDA } \\
\text { growth }\end{array}$ & $\begin{array}{l}\text { Germ- } \\
\text { tube }\end{array}$ & Identification \\
\hline 1 & Throat swab & Purple & $+/-$ & Cream & + & Negative & C. glabrata \\
\hline 2 & Throat swab & No growth & - & Cream & $+1-$ & Negative & C. inconspicua \\
\hline 3 & Mouth ulcer & Green & + & Cream & + & Positive & C. albicans \\
\hline 4 & Throat swab & Green & +++ & Cream & + & Positive & C. albicans \\
\hline 5 & Stool & Purple & +++ & Cream & + & Negative & C. inconspicua \\
\hline 6 & Stool & Purple & + & No growth & - & Negative & C. inconspicua \\
\hline 7 & Stool & Green & $+1-$ & Cream & + & Positive & C. albicans \\
\hline 8 & Throat swab & Green & -+++ & Cream & +++ & Negative & C. albicans \\
\hline 9 & Stool & Green & + & Cream & + & Positive & C. albicans \\
\hline 10 & Stool & Purple & $+1-$ & Cream & $+1-$ & Negative & C. inconspicua \\
\hline 11 & Throat swab & Purple & $+1-$ & Cream & + & Negative & C. inconspicua \\
\hline 12 & Throat swab & Green & +++ & Cream & + & Positive & C. albicans \\
\hline 13 & Throat swab & Green & $+1-$ & Cream & + & Positive & C. albicans \\
\hline 14 & Mouth swab & Green & +++ & Cream & + & Positive & C. albicans \\
\hline 15 & Oesophageal fluid & Green & +++ & Cream & +++ & Positive & C. albicans \\
\hline 16 & Stool & Green & ++ & Cream & + & Positive & C. albicans \\
\hline 17 & Stool & Green & + & Cream & + & Positive & C. albicans \\
\hline 18 & Stool & Purple & $+/-$ & Cream & $+/-$ & Negative & C. inconspicua \\
\hline 19 & Stool & Green & + & Cream & $+1-$ & Positive & C. albicans \\
\hline 20 & Throat swab & Pink & + & Cream & $+1-$ & Negative & C. glabrata \\
\hline 21 & Stool & Green & + & Cream & $+1-$ & Positive & C. albicans \\
\hline
\end{tabular}

-, no growth; +/-, 1-10 colonies; +, 11-40 colonies; ++, 41-100 colonies;,$+++ 101-200$ colonies. 
298 samples

Primary inoculation of swab specimens, $£ 149.00$ labour

(1)

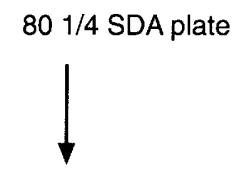

20 yeast culture positives

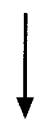

(2)

20 germ-tubes

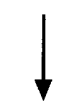

11 positive germ-tubes

$$
\downarrow
$$

$£ 3.50$ cost

(of germ-tube test)

$£ 17.53$ labour

(to set up and read tubes)

(3)

9 API tests

\author{
$£ 21.51$ cost \\ (of API galleries) \\ $£ 6.01$ labour \\ (to set up)
}

Fig. 1. Pathway 1. Steps: 1, culture of swab on to a $1 / 4$ SDA plate, incubated aerobically for $24 \mathrm{~h}$ at $37^{\circ} \mathrm{C} ; 2$, germtube test performed on all yeast isolates; 3, API test performed on all germ-tube-negative isolates.

There was a $100 \%$ correlation of CA with the API test for identification of $C$. albicans whether determined by primary inoculation or follow-up, which confirms other studies $[10,11]$.

We are unable to comment on the sensitivity and specificity of the method for the non- $C$. albicans species, as an unusually high percentage of $C$. inconspicua was isolated. Other studies show good specificity for C. glabrata, C. tropicalis and C. krusei. San-Millan et al. [11] showed $100 \%$ specificity and sensitivity for C. krusei and 93.8\% and 99.1\%, respectively, for $C$. tropicalis. Bernal et al. [10] also showed $99 \%$ and $100 \%$ specificity for $C$. tropicalis and C. krusei, respectively. Odds and colleagues [3] found sensitivities exceeding $99 \%$ for both C. krusei and C. tropicalis.

Two isolates produced negative germ-tube results but were found to be $C$. albicans by biochemical identification. Given the observer variability encountered with the germ-tube test, as well as its lower sensitivity and specificity, CA provides a simple and precise means for rapid identification of C. albicans in the routine laboratory.

The time taken to identify $C$. albicans following pathway 1 was $c .30 \mathrm{~h}$. This is solely dependent on whether the germ-tube test is performed at $24 \mathrm{~h}$ after sample inoculation. The time scale of $30 \mathrm{~h}$ may vary 

Primary inoculation of swab
specimens, $£ 149.00$ labour

(1)
298 samples

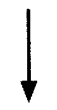

$801 / 4$ SDA plates

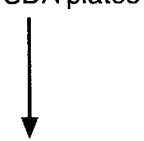

20 yeast culture positives
$£ 15.10 \mathrm{cost}$

(of plates)

£6.35 labour

(to read plates)

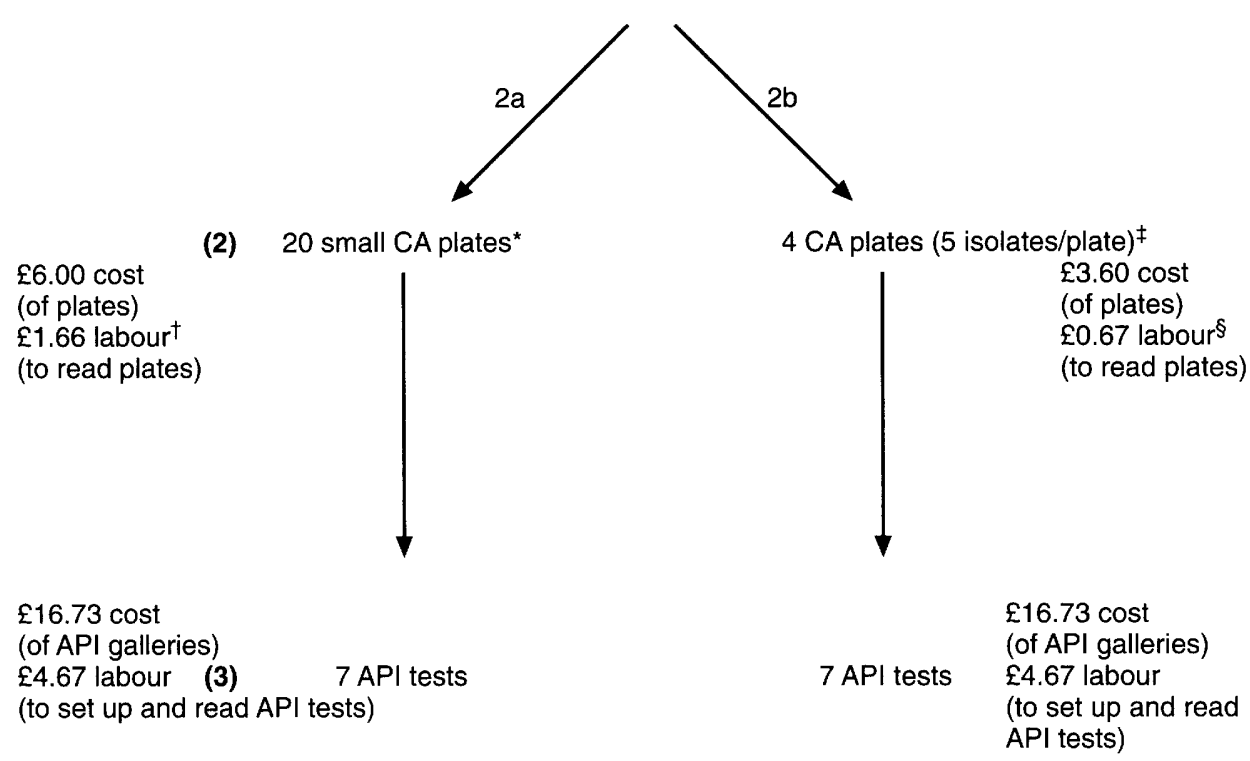

Fig. 2. Pathway 2. Steps: 1, culture swab on to a $1 / 4$ SDA plate, incubated for $24 \mathrm{~h}$ at $37^{\circ} \mathrm{C}$; 2 , culture of yeast isolates on to a CA plate; 3, API test performed on all non-green colony types. ${ }^{*} \mathrm{CA}$ agar set up at 10.00 am and read at $4.00 \mathrm{pm}$. ${ }^{\dagger}$ Based on reading 20 individual plates, c. $30 \mathrm{~s}$ to read a plate; ${ }^{\star}$ Yeast isolates collected and tested on the CA plate twice a week; ${ }^{\S}$ based upon the cost of reading four plates inoculated with the 20 isolates.

according to the time taken for an individual to 'read' a routine batch of specimens, and also if a mixed culture of yeasts is obtained, a pure culture would have to be obtained before any diagnostic test is carried out.

Following pathway 2a, C. albicans could be identified within $30 \mathrm{~h}$ after sample inoculation. The time here is also dependent on whether a pure isolate is cultured initially and whether the yeast isolate is inoculated on to a CA plate $24 \mathrm{~h}$ after primary culture. All isolates producing green colouration within $6 \mathrm{~h}$ were confirmed as $C$. albicans by biochemical identification. Pathway $2 b$ was calculated to be insignificantly less expensive than $2 \mathrm{a}$; however, the time taken to identify the yeast strains isolated following this method could take up to $72 \mathrm{~h}$ longer, because the isolates would have been collected and identified in two batches per week.

Pathway 3 took the shortest time to identify $C$. albicans.
The most economical method is to use CA for identification only (pathway 2). In laboratories with large numbers of isolates, daily inoculation of plates allows C. albicans identification within $30 \mathrm{~h}$, commensurate with current methodology employing germtube production. Additional costs of processing these samples by pathway 3 in our laboratory, compared with conventional methods would be UK $£ 385.71$ per year. The advantages of using this method in some laboratories may outweigh this modest additional cost. Certainly, primary inoculation of blood cultures on to CA (pathway 3) when yeasts are seen on microscopy would gain time where early appropriate therapy is critical.

In conclusion, CHROMagar provides a simple and inexpensive method for the identification of yeasts. This should allow laboratories to introduce identification of mucosal isolates, now increasingly required and recommended [12], with minimal impact on workflows and laboratory budgets. 


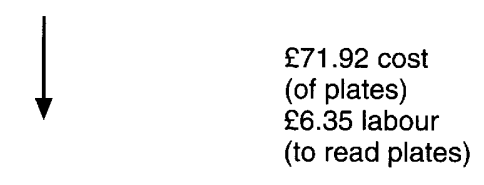

13 green colonies $\quad 7$ non-green colonies

(2)

$7 \mathrm{API}$ tests
$£ 16.73$ cost

(of API galleries)

$£ 4.67$ labour

(to set up and

read $\mathrm{API}$ tests)

Fig. 3. Pathway 3. Steps: 1, culture of swab on to a $1 / 4 \mathrm{CA}$ plate for $24 \mathrm{~h}$ at $37^{\circ} \mathrm{C}$ aerobically; 2 , API test performed on all non-green colony types.

Table 2. Characteristics of pathways 1,2 and 3

\begin{tabular}{|c|c|c|c|c|c|}
\hline Pathway & $\begin{array}{l}\text { Labour cost } \\
(f)\end{array}$ & $\begin{array}{c}\text { Cost of materials } \\
(\mathfrak{f})\end{array}$ & $\begin{array}{l}\text { Total cost } \\
\text { (f) }\end{array}$ & $\begin{array}{l}\text { Minimum time taken } \\
\text { to identify } C \text {. albicans } \\
\text { (h) }\end{array}$ & $\begin{array}{c}\text { Minimum time taken } \\
\text { to identify Candida } \\
\text { spp. }{ }^{*} \text { (h) }\end{array}$ \\
\hline 1 & 178.89 & 40.11 & 219.00 & 30 & 72 \\
\hline $2 a / 2 b$ & $161.68 / 160.69$ & $37.83 / 35.43$ & $199.51 / 196.12$ & $30^{\dagger} / 30^{\ddagger}$ & $72 / 72^{\S}$ \\
\hline 3 & 160.02 & 88.65 & 248.67 & 24 & 72 \\
\hline
\end{tabular}

*Assuming 42-48 h API incubation, i.e., strips read and identification successful after two periods of overnight incubation.

Inoculating plates at 10.00 am allows green colouration to be detected at $4.00 \mathrm{pm}$.

${ }^{\ddagger}$ Some batched isolates will take $102 \mathrm{~h}$.

${ }^{\S}$ Some batched isolates will take $144 \mathrm{~h}$.

We acknowledge the support of Janssen-Cilag Ltd in supplying some of the reagents used in this study.

\section{References}

1. Odds FC. Sabouraud('s) agar. J Med Vet Mycol 1991; 29: 355-359.

2. Beighton D, Ludford R, Clark DT et al. Use of CHROMagar Candida medium for isolation of yeasts from dental samples. $J$ Clin Microbiol 1995; 33: 3025-3027.

3. Odds FC, Bernaerts R. CHROMagar Candida, a new differential isolation medium for presumptive identification of clinically important Candida species. J Clin Microbiol 1994; 32: $1923-1929$.

4. Louwagie B, Surmont I, Verhaegen J, Odds F. Differential and enrichment media for selective culture and recognition of yeast species from clinical material. Eur J Clin Microbiol Infect Dis
1995; 14: 406-411.

5. Pfaller MA, Houston A, Coffmann S. Application of CHROMagar Candida for rapid screening of clinical specimens for Candida albicans, Candida tropicalis, Candida krusei and Candida glabrata (Torulopsis). J Clin Microbiol 1996; 34: 58-61.

6. Odds FC. Candida and candidosis, 2nd edn. London, BailliereTindall. 1988: 68-92.

7. Mackenzie DWR. Serum germ tube production of Candida albicans. J Clin Pathol 1962; 15: 563-565.

8. Welcan UK. Workload measurement system manual, 1992 edn.

9. Baumgartner C, Freydiere A-M, Gille Y. Direct identification and recognition of yeast species from clinical material by using albicans ID and CHROMagar Candida plates. $J$ Clin Microbiol 1996; 34: 454-456.

10. Bernal S, Martin Mazuedos E, Garcia M, Aller Al, Martinez MA, Gutierrez MJ. Evaluation of CHROMagar Candida medium for the isolation and presumptive identification of species of Candida of clinical importance. Diagn Microbiol 
Infect Dis 1996; 24: 201-204.

11. San-Millan R, Ribacoba L, Ponton J, Quindos G. Evaluation of a commercial medium for identification of Candida species. Eur J Clin Microbiol Infect Dis 1996; 15: 153-158.
12. Working Parties of the British Society for Antimicrobial Chemotherapy and the British Society for Medical Mycology 1996. Fungal infections: a survey of laboratory services for diagnosis and treatment. Commun Dis Rep CDR Review 1996; 6: Review no. 5 . 